\title{
A MATHEMATICAL METHOD FOR OPTIMAL INVENTORY POLICIES WITH BACKLOG SALES
}

\author{
Md Abu Helal' ${ }^{1}$, Alain Bensoussan ${ }^{2}$, Viswanath Ramakrishna ${ }^{3}$, Suresh P. Sethi ${ }^{4}$ \\ ${ }^{1}$ Southern Arkansas University - Magnolia, Arkansas, USA \\ ${ }^{2,3,4}$ The University of Texas at Dallas - Richardson, Texas, USA
}

Received 19 November 2020; accepted 20 January 2021

\begin{abstract}
This article is concerned with stochastic inventory control problems with backlog sales in stock-out situations. We examine an infinite horizon model for piecewise linear concave ordering costs. Unlike finite horizons, however, infinite horizons lead to a functional equation for the value function. Such functional equations are solved numerically. Here we give a rigorous theory which explicitly solves this functional equation. We consider both the scenario in which an optimal selection can be made among two suppliers, as well as the scenario in which inventory can be purchased with incremental quantity discounts from a single supplier. We provide conditions that guarantee the optimality of standard $(s, S)$ policy. Moreover, when these conditions fail to hold, we show that an extended four parameter policy is optimal.
\end{abstract}

Keywords: inventory $(s, S)$ policy, backlog sales, general demand distributions, multiple suppliers.

\section{Introduction}

This paper focuses on the widely used ( $s$, $S$ ) inventory policy under general demand distributions and fixed ordering costs. In $(s, S)$ inventory policy, an order is placed when the inventory level falls to the reorder point $s$ or lower so that inventory can be replenished to the level $S$. We consider an infinite-horizon problem with instantaneous deliver systems. Our cost function considers two supplier inventory problems as well as single supplier inventory problems with quantity discounts. In Bensoussan et al. (2020), a similar cost function was studied for lost sales cases. In this paper we consider a backlog sales strategy, i.e., if any excess demand beyond the available stock occurs, then either an emergency order will be placed or the excess amount will be added to the next ordering cycle.

A substantial body of research exists in the discipline of inventory control. The classical inventory $(s, S)$ policy was introduced by Scarf (1960), where he studied a fixed cost and a linear variable unit cost and proved the optimality of $(s, S)$ policy. In 1966 , Veinott introduces a new proof technique which resulted in a similar theorem as that of Scarf (1960), wherein he replaced Scarf's convex cost assumption by a weaker assumption that the negative of expected costs are unimodal. Our problem is concerned with piecewise

${ }^{1}$ Corresponding author: mahelal@saumag.edu 
concave ordering costs. Porteus (1971) was the first to study problems with this kind of cost structure. Later on, Benjaafar et al. (2018) explored this inventory problem with a finite horizon and general demand. They used the notion of generalized $K$-convexity to show its optimality, and conjectured the optimality under horizons which are sufficiently long. We refer the reader to (Chen and Simchi-Levi 2012; Karlin and Fabens, 1960; Singha et al., 2017) for more information on the concave cost function and/or backlog sales. The first reference in particular has an extensive bibliography on the subject.

There is abundant literature available in the area of backlog sales - see, for instance Karlin and Fabens (1960), Beyer et al. (1998), Ozekici and Parlar (1999), Sethi and Cheng (1997), and Song and Zipkin (1993) are mentioned some of them. The problem studied in this paper also has the feature of concave ordering costs. Such ordering costs are frequent in applications. For example, the cost function, when incremental discounting is applied, is represented by a piecewise concave function. We refer the reader to Chen et al. (2005), Huh and Janakiraman (2008), and Song et al. (2009) for more on concave cost functions.

This article corresponds to the $(s, S)$ policy and generalized $(s, S)$ policy with a variety of modifications of the classical work of Scarf and the cost function he considered. Specifically we address two different situations with regards to the cost structure for backlog model. The first one is the classical case involving only one linear segment to illustrate our methodology in proving the optimality of the well known (s, $S$ ) policy. Here we offer a rigorous theory of solving stationary infinite-horizon optimal inventory control problems with general demands and cost parameters satisfying some technical assumptions usual in infinitehorizon settings. It is important to mention that our methodology does not require the notion of $\mathrm{K}$-convexity, introduced in Sethi and Cheng (1997) and used commonly in the literature.

The second situation is the case of two suppliers, first of whom charges a positive fixed ordering cost plus a proportional cost and the second one charges a higher fixed cost plus a lower proportional cost. Here we restrict only an exponential demand. In this case, we show that an extended ( $s$, $S)$ policy, called a $(\sigma, s, \Sigma, S)$ policy, where $\sigma<s \leq \Sigma<S$, is optimal. According to this policy if the beginning inventory is more than $\sigma$ and less or equal to $s$, then order up to $\Sigma$; if it is less or equal to $\sigma$, then order up to $S$; and if it is more than $s$, then do not order. Moreover, we obtain these four parameters explicitly due to the simplicity of the exponential demand.

We formulate the Bellman equation which in the infinite horizon case becomes a functional equation. We provide a rigorous mathematical theory to solve this functional equation and show that the optimality of the $(s, S)$ policy for inventory control problems with piecewise linear concave ordering costs. The theory is constructive and does not rely on the notion of $K$-convexity. We allow demand distributions which are more general than those considered by Porteus (1971). Furthermore, we prove the conjecture of Benjaafar et al. (2018) about the infinitehorizon setting. While we examine only the case of dual suppliers in this article, our methodology is more general and can be used to extend the results to situations with an arbitrary number of suppliers. 


\section{Formulation of Problem}

The generalization of the cost structure concerns the ordering cost, which is now the concave function $c(v), v>0$, given by the formula:

$c(v)=\min _{i=1, \ldots, n}\left(K_{i}+c_{i} v\right)$,

With the conditions $0 \leq K_{1}<K_{2}<\cdots<K_{n}, c_{1}>c_{2}>\cdots>c_{n} \geq 0$, where $v$ is the total amount of order, $c_{\mathrm{i}}$ and $K_{\mathrm{i}}$ is the per unit cost and fixed cost respectively of the $i^{\text {th }}$ supplier. We begin with the assumption that our cost function is piecewise linear concave comprised of two pieces, See in the Fig. 1 . To provide a practical justification for choosing $n=2$, we claim that the structure of equation (1) applies not just to the problem of choosing among several suppliers, but can also represent the situation of rebates for large quantities in the case of a single supplier. Assume that $n=2$ in equation (1), and let $\epsilon=\left(K_{2}-K_{1}\right) /\left(c_{1}-c_{2}\right)$. From here, one can readily verify that

$c(v)=K_{1} \mathbb{1}_{v>0}+\left(c_{1}-c_{2}\right) v \mathbb{1}_{v<\epsilon}+c_{2} v+\left(c_{1}-c_{2}\right) e \mathbb{1}_{v \geq \epsilon}$.

If the volume is greater than $\epsilon$, we observe that the variable ordering cost $c_{1}$ will reduce to $c_{2}$. Here, as usual $\mathbb{1}_{v}$ is an indicator function.

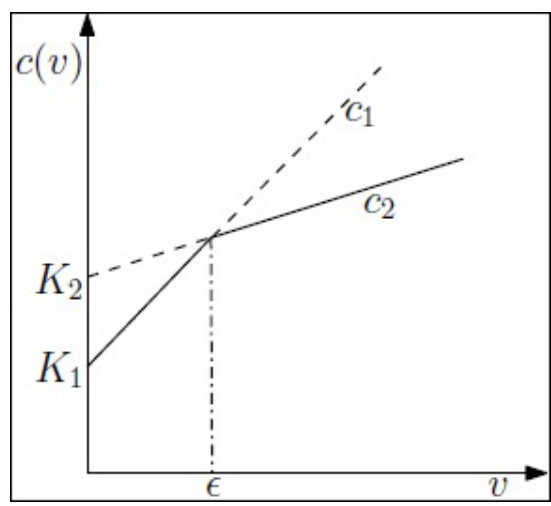

Fig. 1.

The Graph of Cost Function given in Eq. (1) for $n=2$

Denote by $x$ the initial inventory and let $y$ denote the inventory once the order has been received. We denote the generic demand by $D$. $D$ is a random variable with $f(\xi)$ as its probability density function. The $F(x)=\int_{0}^{x} f(\xi) d \xi$ is the cumulative distribution function of $D$. We note that the complement of $F(x)$ is $\bar{F}(x)=1-F(x)$. We denote with $u(x)$ the expected value of the discounted costs for an inventory problem. Then, $u(x)$ satisfies the following Bellman: 


$$
\begin{aligned}
u(x)= & h x^{+}+p x^{-}+\inf _{y \geq x}\left\{K_{1} \mathbb{1}_{y>x}+\left(c_{1}-c_{2}\right)(y-x) \mathbb{D}_{y-x<\epsilon}+\left(c_{2}(y-x)+\left(c_{1}-c_{2}\right) \varepsilon\right) \mathbb{I}_{y-x \geq \epsilon}\right. \\
& +\alpha E u(y-D)\}, x>0 .
\end{aligned}
$$

Functional equation where $h$ and $p$ represents the holding cost and penalty cost respectively. We apply the transformation:

$$
u(x)=h x^{+}+p x^{-}-c_{2} x+H(x)+\rho
$$

to the preceding equation, where $\rho$ is an arbitrary constant. Substituting eq. (4) into eq. (3) results in the following equation for $H(x)$ :

$H(x)+\rho(1-\alpha)=\inf _{y \geq x}\left\{K_{1}+\left(c_{1}-c_{2}\right)(y-x) \mathbb{D}_{y-x<\epsilon}+\left(c_{1}-c_{2}\right) \epsilon \mathbb{1}_{y-x \geq \epsilon}+g(y)+\alpha E H((y-D))\right\}$

Where,

$g(y)=c_{2}(1-\alpha) y+\alpha h E(y-D)^{+}+\alpha p E(y-D)^{-}+\alpha c_{2} D$

The idea is to find an optimal policy in order to minimize the expected total discounted cost or in other words, to achieve the minimum on the right hand side of eq. (5).

\subsection{Primary Discussion}

The above described problem is an extension of the following classical problem, corresponding to $\epsilon=0$. We write $K_{1}=K_{2}=K$ and $c_{1}=c_{2}=c$, then eq. (5) specializing in:

$H(x)+\rho(1-\alpha)=\inf _{y \geq x}\left\{K+g(y)+\alpha E H\left((y-D)^{+}\right)\right\}$

which leads to the classical $(s, S)$ policy solution. We describe briefly how to proceed in this classical case, to foresee the steps for the extension to (5). The solution is described as follows. For any $s>0$, take $\rho(1-\alpha)=g(s)$ and solve for $H_{s}(x)$, the solution of:

$\begin{cases}H_{s}(x)=g(x)-g(s)+\alpha E H_{s}(x-D), & \text { if } x>s, \\ H_{s}(x)=0, & \text { if } x \leq s .\end{cases}$

Since $g(x)$ is a convex function, $H_{s}(x)$ attains its minimum at a point (if there are several points we will take the smallest one). We define the minimum point to be $S$ so that:

$\mathrm{H}_{s}(\mathrm{~S})=\inf _{\mathrm{y}>s} \mathrm{H}_{s}(\mathrm{y})$

Then define $s$ so that:

$0=\mathrm{K}+\inf _{\mathrm{y} \geq \mathrm{s}} \mathrm{H}_{\mathrm{s}}(\mathrm{y})$. 
A unique $s$ can be defined, to which one associates $S=S(s)$. This is the well known $(s, S)$ policy.

\subsection{Rewriting Bellman Equation}

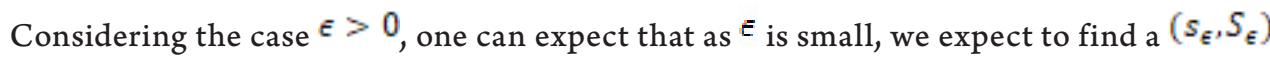
policy that is optimal. To do so, we begin by rewriting the Bellman functional equation defined in eq. (5) as follows:

$$
\begin{aligned}
H(x)+\rho(1-\alpha) & =\min \{g(x)+\alpha E H((x-D)) \\
& K_{1}+\inf _{x<y \leq x+\epsilon}\left(\left(c_{1}-c_{2}\right)(y-x)+g(y)+\alpha E H((y-D))\right) \\
& \left.K_{2}+\inf _{y>x+\epsilon}(g(y)+\alpha E H((y-D)))\right\}
\end{aligned}
$$

To see that this reduces to a $(s, S)$ policy of two parameters, we show that $K_{1}+\inf _{x<y \leq x+\epsilon}\left(\left(c_{1}-c_{2}\right)(y-x)+g(y)+\alpha E H((y-D))\right)$ can be eliminated from eq. (11) as follows:

$H(x)+\rho(1-\alpha)=\min \left\{g(x)+\alpha E H(x-D), K_{2}+\inf _{y>x+\epsilon}(g(y)+\alpha E H(y-D))\right\}$.

In the next section we set out the sufficient conditions for optimality and show that there exists a pair $\left(s_{\epsilon}, S_{\epsilon}\right)$ that satisfy eq. (12) and hence $\left(s_{\epsilon}, S_{\epsilon}\right)$ policy is optimal.

\section{Optimality of $\left(s_{\epsilon}, S_{\epsilon}\right)$ Policy}

Analogous to the case where $\epsilon=0$, for any $s>0$, we take $\rho(1-\alpha)=g(s)$ and solve the problem:

$$
\begin{cases}H_{s}(x)=g(x)-g(s)+\alpha E H_{s}(x-D), & \text { for } x>s, \\ H_{s}(x)=0, & \text { for } x \leq s .\end{cases}
$$

To obtain a solution to the above equation, we fix $s$ such that: 
$\inf _{y>x+\epsilon}(g(y)+\alpha E H(y-D))=-K_{2}$

We take the derivative of (13) with respect to $x$ and obtain:

$\begin{cases}H_{s}{ }^{\prime}(x)=g^{\prime}(x)+\alpha E H_{s}{ }^{\prime}(x-D), & \text { for } x>s, \\ H_{s}{ }^{\prime}(x)=0, & \text { for } x \leq s .\end{cases}$

As $g^{\prime}(x)$ is bounded, we have a contraction problem. Therefore, by the Contraction Mapping Theorem, we know that the function has a unique fixed point. Accordingly, the above problem has exactly one solution. Since $H_{s}{ }^{\prime}(x-0)=0_{\text {but }} H_{s}{ }^{\prime}(x+0)=g^{\prime}(x)$, we have that $H_{s}{ }^{\prime}(x)$ is not continuous at $s$. But we can make $H_{s}{ }^{\prime}(x)$ continuous by letting $s=\bar{s}$ such that $g^{\prime}(\bar{s})=0$, which implies that $\alpha(h+p) F(\bar{s})=\alpha p-c_{2}(1-\alpha)$, and therefore, $F(\bar{s})=\frac{\alpha p-c_{2}(1-\alpha)}{\alpha(h+p)}$

From equation (6), we observe that:

$g^{\prime}(y)=c_{2}(1-\alpha)-\alpha p+\alpha(h+p) F(y) ;$ for $y>0$,

$g^{\prime}(y)=c_{2}(1-\alpha)-\alpha p ; \quad$ for $y<0$.

Next, we assume that

$\alpha\left(p+c_{2}\right)>c_{2}$

Then, since $p>c_{2}$, the function $g^{\prime}(x)$ must be increasing from $c_{2}(1-\alpha)-\alpha p$ to $c_{2}(1-\alpha)+\alpha h$. Therefore, $c_{2}(1-\alpha)-\alpha p<g^{\prime}(x)<c_{2}(1-\alpha)+\alpha h$. From equation (15) it follows that:

$\frac{c_{2}(1-\alpha)-\alpha p}{1-\alpha} \leq H_{s}^{\prime}(x) \leq \frac{c_{2}(1-\alpha)+\alpha h}{1-\alpha}$ 
Moreover, $H_{s}^{\prime}(\infty)=\frac{c_{2}(1-\alpha)+\alpha n}{1-\alpha}$. Therefore, the function $H_{s}(x)$ is defined by:

$H_{s}(x)=\int_{s}^{x} H_{s}^{\prime}(\zeta) d \zeta, x \geq s$

$H_{s}(x)$ goes to $\infty$ as $x$ goes to $\infty$, and since $H_{s}(x)$ is continuous, we have that the function attains its minimum on the interval $[s, \infty)$. Therefore, we claim that there exists a unique $S(s)$ such that:

$H_{s}(s(s))=\inf _{y \geq s} H_{s}(y)$

We will necessarily have that $(s) \geq \bar{s} \forall s<\bar{s}$, for otherwise if $s>\bar{s}$ then we would have that $S(s)=s$. We want to find $s_{\varepsilon}$ such that $s_{\varepsilon}$ is a solution of

$K_{2}+\inf _{y \geq s_{\varepsilon}+\epsilon} H_{s_{\varepsilon}}(y)=0$

Of course, $s_{\epsilon}+\epsilon<\bar{s}$, which implies that

$\epsilon<\bar{s}$.

This suggests that $\epsilon$ is small. From the inequality $s_{\epsilon}+\epsilon<\bar{s}$, the definition of $\bar{s}$, and equation (15), we can conclude that $H^{\prime}{ }_{s_{\varepsilon}}(y)<0$, if $y \in\left[s_{\varepsilon}, s_{\varepsilon}+\varepsilon\right]$. Therefore, $y \rightarrow H_{s_{\varepsilon}}(y)$ is decreasing on $\left[s_{\epsilon}, s_{\epsilon}+\epsilon\right]$. Hence:

$\inf _{y \in\left[s_{\varepsilon}, s_{\varepsilon}+\varepsilon\right]} H_{s_{\varepsilon}}(y)=H_{s_{\varepsilon}}\left(s_{\varepsilon}+\epsilon\right) \geq \inf _{y \geq s_{\varepsilon}+\varepsilon} H_{s_{\varepsilon}}(y)$

So we can write eq. (22) as:

$K_{2}+\inf _{y \geq s_{\varepsilon}} H_{s_{\varepsilon}}(y)=0$

Lemma 1: We claim that

$\mathrm{s} \mapsto \inf _{\mathrm{y} \geq \mathrm{s}} \mathrm{H}_{\mathrm{s}}(\mathrm{y})=\mathrm{H}_{\mathrm{s}}(\mathrm{s}(\mathrm{s}))$

is increasing on $(-\infty, \bar{s})$

Proof: Indeed, writing eq. (15) as:

$H_{s}(x)=g(x)-g(s)+\alpha \int_{0}^{x-s} H_{s}(x-\xi) f(\xi) d \xi, x>s$

we see that $\frac{\partial H_{s}(x)}{\partial s}, x>s$, is a solution to the equation 
$\frac{\partial H_{s}(x)}{\partial s}=-g^{\prime}(s)+\alpha \int_{0}^{x-s} \frac{\partial H_{s}(x-\xi)}{\partial s} f(\xi) d \xi, x>s$

For $s<\bar{s}$, we have that $g^{\prime}(s)<0$. Therefore, $\frac{\partial H_{s}(x)}{\partial s}>0, x>s$.

Nextinf ${ }_{y>s} H_{s}(y)=H_{s}(S(s))$ and $\frac{d}{d s} H_{s}(S(s))=\frac{\partial H_{s}(S(s))}{\partial s}$ because of the optimality of $S(s)$. Moreover, $H_{-\infty}^{\prime}(x)=g^{\prime}(x)+\alpha E H_{-\infty}^{\prime}(x-D)$ and

$H_{-\infty}(x)=\int_{-\infty}^{x} H_{-\infty}^{\prime}(\xi) d \xi=-\infty, \forall x$

This result is true because $g^{\prime}(x)$ is a constant $\forall x<0$. Finally, since inf $\operatorname{ins}_{s} H_{s}(S(s))$ is increasing on $s$ and it is 0 at $\bar{s}$, the integral evaluates to $-\infty$ when $x=-\infty$. This proves the property asserted in (26). So, it is clear that there exists a single $s_{\epsilon}$ for which $\inf _{y \geq s_{\varepsilon}} H_{s_{\varepsilon}}(y)=-K_{2}$. Now we can state the following:

Proposition 2: Assume that inequalities (18) and (23) hold. Then there exists a unique $\mathrm{s}_{\varepsilon}<\bar{s}-\epsilon$ which is a solution of eq. (25) with $\mathrm{H}_{s}(\mathrm{x})$ uniquely defined by eq. (13). The solution of equation (13) is given by eq. (20).

We want now to check that the solution $H_{s_{\varepsilon}}(x)$ is a solution to our original problem, (11), with $\rho(1-\alpha)=g\left(s_{\epsilon}\right)$. Denoting $S_{\epsilon}=S\left(s_{\epsilon}\right)$, this defines the $\left(s_{\epsilon}, S_{\epsilon}\right)$ policy that we will show to be optimal. Doing so will require use of the following theorem.

Theorem 3: In addition to the assumptions of Proposition 2, assume that

$\alpha p<c_{1}(1-\alpha)$

Then the function $\mathrm{H}_{s_{\varepsilon}}(\mathrm{x})$ is a solution to equation (11), with $\rho(1-\alpha)=g\left(s_{\epsilon}\right)$.

Proof: Lacking the assumption that (16) holds, we can still prove that $H_{s_{\varepsilon}}(x)$ is solution of (12). That is, we can show that:

$H_{s_{\varepsilon}}(x)=\min \left(g(x)-g\left(s_{\varepsilon}\right)+\alpha E H_{s_{\varepsilon}}(x-D), K_{2}+\inf _{y>x+\varepsilon}\left(g(y)-g\left(s_{\varepsilon}\right)+\alpha E H_{s_{\varepsilon}}(y-D)\right)\right)$

Now assume that $x \leq s_{\xi}$. Then $H_{s_{\varepsilon}}(x)=0$ and $g(x)-g\left(s_{\varepsilon}\right)+\alpha E H_{s_{\varepsilon}}(x-D)=g(x)-g\left(s_{\xi}\right)>0$, since $g(x)$ decreases whenever $x<\bar{s}$. Next, if $x+\epsilon<s_{\xi}$, then:

$$
\begin{aligned}
\inf _{y>x+\varepsilon}\left(g(y)-g\left(s_{\xi}\right)+\alpha E H_{s_{\varepsilon}}(y-D)\right. & =\min \left(\operatorname { i n f } _ { x + \varepsilon < y < s _ { \varepsilon } } \left(g(y)-g\left(s_{\epsilon}\right),\right.\right. \\
& \inf _{s_{\varepsilon} \leq y}\left(g(y)-g\left(s_{\xi}\right)+\alpha E H_{s_{\varepsilon}}(y-D)\right) \\
& =\min \left(\inf _{x+\varepsilon<y<s_{\varepsilon}}\left(g(y)-g\left(s_{\varepsilon}\right), \inf _{s_{\varepsilon} \leq y} H_{s_{\varepsilon}}(y)=-K_{2}\right)\right.
\end{aligned}
$$


since inf $\operatorname{in}_{x+\varepsilon<y<s_{\varepsilon}}\left(g(y)-g\left(s_{\epsilon}\right)>0\right.$. Therefore, the right hand side of (31) is 0 , which is indeed the value of $H_{s_{\varepsilon}}(x)$ when $x \leq s_{\varepsilon}$. Accordingly, $H_{s_{\varepsilon}}(x)$ is solution of (31) when $x+\epsilon \leq s_{\varepsilon}$. When $s_{\epsilon} \geq x>s_{\epsilon}-\epsilon$

$\inf _{y>x+\varepsilon}\left(g(y)-g\left(s_{\varepsilon}\right)+\alpha E H_{s_{\varepsilon}}\left((y-D)^{+}\right)\right)=\inf _{y>x+\varepsilon} H_{s_{\varepsilon}}(y)=H_{s_{\varepsilon}}\left(S_{\varepsilon}\right)=\inf _{y \geq s_{\varepsilon}} H_{s_{\varepsilon}}(y)=-K_{2}$

Thus, $H_{s_{\varepsilon}}(x)$ is a solution of (31) when $x \leq s_{\xi}$. If $x>s_{\varepsilon}$, then (31) implies that:

$H_{s_{\varepsilon}}(x)=\min \left(H_{s_{\varepsilon}}(x), K_{2}+\inf _{y>x+\varepsilon} H_{s_{\varepsilon}}(y)\right)$

To check (34) we must prove that:

$H_{s_{\varepsilon}}(x) \leq K_{2}+H_{s_{\varepsilon}}(y), \forall y>x+\epsilon$

But we have:

$H_{s_{\varepsilon}}(x) \leq K_{2}+H_{s_{\varepsilon}}(y), \forall y>x$

Which is a classical result. For more details we refer the reader to the similar proof in Bensoussan (2011), when the case is $\epsilon=0$.

So far we have proven that $H_{s_{\varepsilon}}(x)$ is a solution of (31) which means that it is also a solution of equation (12) with $\rho(1-\alpha)=g\left(s_{\epsilon}\right)$. The assumption of (30) was not needed for this.

To prove that $H_{s_{\varepsilon}}(x)$ is a solution of equation (11), it remains to be proven that:

$H_{s_{\varepsilon}}(x) \leq K_{1}+\inf _{x<y \leq x+\epsilon}\left(\left(c_{1}-c_{2}\right)(y-x)+g(y)-g\left(s_{\epsilon}\right)+\alpha E H_{s_{\varepsilon}}(y-D)\right)$

If $x+\epsilon \leq s_{\epsilon}$, then equation (37) can be rewritten as:

$0 \leq K_{1}+\inf _{x<y \leq x+\epsilon}\left(\left(c_{1}-c_{2}\right)(y-x)+g(y)-g\left(s_{\varepsilon}\right)\right)$

Which we know to be true because $g(y)-g\left(s_{\epsilon}\right)>0$ for $y \leq s_{\varepsilon}$. So we may assume $s_{\epsilon}<x+\epsilon$. If $x \geq s_{\epsilon}$ then eq. (37) means that:

$H_{s_{\varepsilon}}(x) \leq K_{1}+\inf _{x<y \leq x+\varepsilon}\left(\left(c_{1}-c_{2}\right)(y-x)+H_{s_{\varepsilon}}(y)\right)=K_{1}+H_{s_{\varepsilon}}(x)$ 
On account of assumption (30) and the fact that estimate (19) means that the function $\left(c_{1}-c_{2}\right) y+H_{s_{\varepsilon}}(y)$ is monotone increasing when $y>s_{\varepsilon}$. So, (37) is true if $x \leq s_{\varepsilon}-\epsilon$ or $x \geq s_{\epsilon}$. Finally, if $s_{\epsilon}-\epsilon<x<s_{\epsilon}$, (37) means that:

$0 \leq K_{1}+\min \left(\inf _{x<y \leq s_{\varepsilon}}\left(\left(c_{1}-c_{2}\right)(y-x)+g(y)-g\left(s_{\varepsilon}\right)\right), \inf _{s_{\varepsilon}<y \leq x+\varepsilon}\left(\left(c_{1}-c_{2}\right)(y-x)+H_{s_{\varepsilon}}(y)\right)\right)$

Which previous arguments have shown to be true. This completes our proof.

The significance of equation (30) is briefly summarized in the following remark:

Remark: Assumption (30) serves to ensure that a standard $(s, S)$ policy will be optimal, and requires the penalty cost to be small. Assumption (30) ensures that $H_{s_{\varepsilon}}(x)$ is a solution of eq. (11). Moreover, since (30) implies that $p \leq c_{1}$, we have that $c_{2}<p \leq c_{1}$. Therefore, any purchase from supplier 1 will $\operatorname{cost} c_{1}$ in addition to any fixed cost. On the other hand, electing to not meet the demand will only $\operatorname{cost} p$, which is less than $c_{1}$. In such a situation, orders will only be placed with the second supplier. Furthermore, if we make $\epsilon$ small solely by increasing $K_{1}$ and/or $c_{1}$, then the optimal policy is exactly the $(s, S)$ policy of the classical problem with only the second supplier. This will hold for every $\epsilon$ as long as it is small and (30) continues to hold. Therefore, we have shown that the $\left(s_{\varepsilon}, S_{\epsilon}\right)$ policy is optimal.

On the other hand when $p>c_{1}$, which necessarily means that (30) is violated, we will show in the next section that an extended $(s, S)$ policy is optimal.

\section{Extended $(s, S)$ Policy}

The result of Section 3 relied heavily upon the assumption (30). If assumption (30) is not satisfied, we cannot guarantee that a standard $(s, S)$ policy is optimal. In this case, we look for an extended $(s, S)$ policy. In this section, we assume the demand density to be exponential and we will explicitly show that a four parameter $(\sigma, s, \Sigma, S)$ policy is optimal.

\subsection{Problem Formulation}

At this stage, we look for a solution of equation (11) of the following form: there are two numbers $\sigma$, s with $0<\sigma<s$ and the function $H(x)$ satisfies:

$$
\begin{cases}H(x)=H(\sigma), & \forall x \leq \sigma \\ H(x)=H(s)+\left(c_{1}-c_{2}\right)(s-x), & \sigma<x \leq s \\ H(x)=g(x)-g(s)+\alpha E H(x-D), & x>s .\end{cases}
$$

The function $H(x)$ is continuous. Therefore, the values of $H(s)$ and $H(\sigma)$ are given by the above conditions. We first have from the first two parts of equation (41), that

$$
H(\sigma)=H(s)+\left(c_{1}-c_{2}\right)(s-\sigma) \text {. }
$$


From the third segment we get:

$H(s)=\alpha E H(s-D)$.

Next we integrate equation (43) and plugging the expressions of first two segments of (41) into (43). After all that, simplify equation (43), and it becomes

$H(s)=\frac{\alpha}{1-\alpha}\left(c_{1}-c_{2}\right) \int_{0}^{s-\sigma} \bar{F}(\xi) d \xi$

So, for $x>s$, the function $H(x)$ is solution of the integral equation:

$$
\begin{aligned}
H(x)= & \alpha \int_{0}^{x-s} H(x-\xi) f(\xi) d \xi+g(x)-g(s)+ \\
& \alpha\left(c_{1}-c_{2}\right)\left[\int_{0}^{s-\sigma} \bar{F}(x-s+\eta) d \eta+\frac{\alpha}{1-\alpha} \bar{F}(x-s) \int_{0}^{s-\sigma} \bar{F}(\xi) d \xi\right]
\end{aligned}
$$

Clearly, the function $H(x)$ is completely defined once the constants $\sigma, s$ are fixed. To define $\sigma$ and $s$, we impose the following two conditions:

$$
\begin{aligned}
& H(s)=K_{1}+\inf _{s<y \leq s+\epsilon}\left(\left(c_{1}-c_{2}\right)(y-s)+H(y)\right) \\
& H(\sigma)=K_{2}+\inf _{y>s}(y)
\end{aligned}
$$

Next, we need to show that equations (46) and (47) in the unknowns $0<\sigma<s$ define them completely, and that the corresponding function $H(x)$ solves equation (11), with $\rho(1-\alpha)=g(s)$. We then associate to the pair $\sigma, s$ the numbers $\Sigma, S$ which realize the infimum in the right hand side of (46), (47) respectively. The four parameter $\sigma_{\epsilon}<s_{\epsilon} \leq \Sigma_{\epsilon}<S_{\epsilon}$ will be the extended policy solving the problem.

Now to study the equation (45) we first define the derivative in the form of:

$H^{\prime}(x)=\alpha \int_{0}^{x-s} H^{\prime}(x-\xi) f(\xi) d \xi+g^{\prime}(x)+\alpha\left(c_{1}-c_{2}\right)(\bar{F}(x-\sigma)-\bar{F}(x-s)), x>s$

Since we assume $f(\zeta)=\lambda e^{-\lambda \zeta}$, the integral equation (48) becomes:

$H^{\prime}(x)=\lambda \alpha \int_{0}^{x-s} H^{\prime}(x-\xi) e^{-\lambda \xi} d \xi+c_{2}+\alpha\left(h-c_{2}\right)+e^{-\lambda x}\left[-\alpha(h+p)+\alpha\left(c_{1}-c_{2}\right)\left(e^{\lambda \sigma}-e^{\lambda s}\right)\right]$

It has an explicit solution given by:

$H^{\prime}(x)=c_{2}+\frac{\alpha \hat{h}}{1-\alpha}+e^{-\lambda(1-\alpha)(x-s)}\left[\alpha\left(c_{1}-c_{2}\right) e^{-\lambda(s-\sigma)}-\alpha(h+p) e^{-\lambda s}-\alpha\left(c_{1}+\frac{\alpha \hat{h}}{1-\alpha}\right)\right]$ 
This equation has one and only one solution, on $[s,+\infty)$. The next step is to find the function $s=s(\sigma)$. For fixed $\sigma$, we define $s(\sigma)>\sigma$, by solving the algebraic equation:

$\frac{\alpha}{1-\alpha}\left(c_{1}-c_{2}\right) \int_{0}^{s-\sigma} \bar{F}(\xi) d \xi=K_{1}+\inf _{s<y \leq s+\varepsilon}\left(\left(c_{1}-c_{2}\right)(y-s)+H_{\sigma s}(y)\right)$

and finally, we search for $\sigma$ as the solution of the algebraic equation:

$\left(c_{1}-c_{2}\right)(s(\sigma)-\sigma)+\frac{\alpha}{1-\alpha}\left(c_{1}-c_{2}\right) \int_{0}^{s(\sigma)-\sigma} \bar{F}(\xi) d \xi=K_{2}+\inf _{y>s(\sigma)} H_{\sigma, s(\sigma)}(y)$

If we are able to solve equation (51) and (52), then we need to prove that the $H(x)$ satisfies:

$$
\begin{aligned}
H(x) & =\min \{g(x)-g(s)+\alpha E H((x-D)), \\
& K_{1}+\inf _{x<y \leq x+\varepsilon}\left(\left(c_{1}-c_{2}\right)(y-x)+g(y)-g(s)+\alpha E H((y-D))\right), \\
& \left.K_{2}+\inf _{y>x+\varepsilon}(g(y)-g(s)+\alpha E H((y-D)))\right\}
\end{aligned}
$$

to guarantee that $H(x)$ is the solution of eq. (5).

\subsection{Preliminary Calculation}

First, we need to find the function $s(\sigma)$ by solving equation (51). We expect that the infimum on the right-hand side of equation (51) is attained at a point $\Sigma$ inside the interval $(s, s+\epsilon)$. Therefore, we have $H^{\prime}(\Sigma)+c_{1}-c_{2}=0$, which along with equation (50) yields

$$
e^{\lambda(1-\alpha)(\Sigma-s)}=\frac{\alpha(h+p) e^{-\lambda s}-\alpha\left(c_{1}-c_{2}\right) e^{-\lambda(s-\alpha)}+\alpha\left(c_{1}+\frac{a h}{1-\alpha}\right)}{c_{1}+\frac{a h}{1-\alpha}}
$$

Also eq. (51) becomes to $0=K_{1}+\int_{s}^{E}\left(c_{1}-c_{2}+H^{\prime}(x)\right) d x$, which yields:

$$
\begin{aligned}
& \lambda(1-\alpha)\left[K_{1}+\left(c_{1}+\frac{\alpha h}{1-\alpha}\right)(\Sigma-s)\right]= \\
& \left(1-e^{-\lambda(1-\alpha)(\Sigma-s)}\right)\left[\alpha(h+p) e^{-\lambda s}-\alpha\left(c_{1}-c_{2}\right) e^{-\lambda(s-\sigma)}+\alpha\left(c_{1}+\frac{\alpha h}{1-\alpha}\right)\right]
\end{aligned}
$$

Eq. (54) and (55) give $s$ and $\Sigma$ as functions of $\sigma$. For convenient we define:

$X=X(\sigma, s)=\frac{\alpha(h+p) e^{-\lambda s}-\alpha\left(c_{1}-c_{2}\right) e^{-\lambda(s-\sigma)}}{c_{1}+\frac{\alpha h}{1-\alpha}}$ 
Then, from (54) and (55) we find:

$X=\log (X+\alpha)+(1-\alpha)\left(1+\frac{k_{1} \lambda}{c_{1}+\frac{a h}{1-\alpha}}\right)$

It has a unique solution $X_{0}>1-\alpha$, and this is a fixed constant independent of $\sigma$. Therefore, we obtain $s=s(\sigma)$ by:

$e^{\lambda s}=\frac{\alpha(h+p)-\alpha\left(c_{1}-c_{2}\right) e^{\lambda \sigma}}{x_{0}\left(c_{1}+\frac{a h}{1-\alpha}\right)}$

We naturally need $\sigma$ sufficiently small. Specifically, we need $\sigma \leq \bar{s}_{\epsilon}$ with:

$e^{\lambda \bar{s}_{\varepsilon}}=\frac{\alpha(h+p)}{x_{0}\left(c_{1}+\frac{a h h}{1-\alpha}\right)+\alpha\left(c_{1}-c_{2}\right)}$.

In order to get $\bar{s}_{\varepsilon}>0$, we need the condition:

$\alpha(h+p)>X_{0}\left(c_{1}+\frac{\alpha h}{1-\alpha}\right)$

and $s\left(\bar{s}_{\epsilon}\right)=\bar{s}_{\varepsilon}$. We have also $s(0)=\bar{s}_{\epsilon}^{*}$ with:

$e^{\lambda \vec{s}_{\varepsilon}^{*}}=\frac{\alpha\left(h+p-c_{1}+c_{2}\right)}{x_{0}\left(c_{1}+\frac{a h}{1-\alpha}\right)}$.

Assumption (60) implies immediately $\bar{s}_{\epsilon}<\bar{s}_{\epsilon}^{*}$. Recall the definition of $\bar{s}$, that is $g^{\prime}(\bar{s})=0$, to obtain

$e^{\lambda \bar{s}}=\frac{\alpha(h+p)}{c_{2}+\alpha\left(h-c_{2}\right)}$.

In view of $X_{0}>1-\alpha$, comparing equations (61) and (62) yields $\bar{s}_{\epsilon}^{*}<\bar{s}$. Summarizing we have:

Proposition 4: Assume (60), i.e., $\alpha(h+p)>X_{0}\left(c_{1}+\frac{a h}{1-\alpha}\right)$. Then for $\sigma \in\left[0, \bar{s}_{e}\right] s(\sigma)$ is uniquely defined by (58), i.e., $e^{\lambda s}=\frac{\alpha(h+p)-\alpha\left(c_{1}-c_{2}\right) e^{\lambda a}}{x_{0}\left(c_{1}+\frac{\alpha h}{1-\alpha}\right)}$, and it decreases from $s(0)=\bar{s}_{\epsilon}^{*}$ to $s\left(\bar{s}_{\epsilon}\right)=\bar{s}_{\epsilon}$. We further have $\bar{s}_{\epsilon}<\bar{s}_{\epsilon}^{*}<\bar{s}$.

Next, $\Sigma(\sigma)$ is defined by (54), which reads as:

$\Sigma(\sigma)-s(\sigma)=\frac{\log \left(x_{0}+\alpha\right)}{\lambda(1-\alpha)}$ 
We can also define $S(\sigma)$ by the condition $H^{\prime}(S)=0$. From $(50)$ and (56), we have

$H^{\prime}(x)=c_{2}+\frac{\alpha \hat{h}}{1-\alpha}-\left(X_{0}+\alpha\right)\left(c_{1}+\frac{\alpha \hat{h}}{1-\alpha}\right) e^{-\lambda(1-\alpha)(x-s)}$

Therefore,

$S(\sigma)-s(\sigma)=\frac{\log \left[\left(x_{0}+\alpha\right) \frac{c_{1}+\frac{a h}{a-\hbar}}{c_{2}+\frac{a h}{1-\alpha}}\right]}{\lambda(1-\alpha)}$

Looking at the form of (49) and noting $\sigma<s$, we can assert

$H^{\prime}(x) \leq \lambda \alpha \int_{0}^{x-s} H^{\prime}(x-\xi) e^{-\lambda \xi} d \xi+c_{2}+\alpha\left(h-c_{2}\right)-e^{-\lambda x}(\alpha(h+p))$

For $s<x<\bar{s}$, we have $c_{2}+\alpha\left(h-c_{2}\right)-e^{-\lambda x}(\alpha(h+p))<0$. Hence,

$H^{\prime}(x) \leq \lambda \alpha \int_{0}^{x-s} H^{\prime}(x-\xi) e^{-\lambda \xi} d \xi, s<x<\bar{s}$

Also from eq. (56):

$H^{\prime}(s+0)=c_{2}+\alpha\left(h-c_{1}\right)-X_{0}\left(c_{1}+\frac{\alpha h}{1-\alpha}\right)<-\left(c_{1}-c_{2}\right)$

From (67) and (68), we see that:

$H^{\prime}(x)<0, s<x<\bar{s}$

which implies necessarily $S(\sigma)>\bar{s}$. Calling $\tilde{H}^{\prime}(x)=H(x)+c_{1}-c_{2}$, we can write the equation for $\tilde{H}^{\prime}(x)$ as

$\stackrel{\mathscr{H}}{\tilde{H}}(x)=\lambda \alpha \int_{0}^{x-s} \tilde{H}^{\prime}(x-\xi) e^{-\lambda \xi} d \xi+c_{1}+\alpha\left(h-c_{1}\right)+e^{-\lambda x}\left[-\alpha(h+p)+\alpha\left(c_{1}-c_{2}\right) e^{\lambda \sigma}\right]$

Hence, for $x>s$, we have:

$\stackrel{\prime}{\tilde{H}}(x) \leq \lambda \alpha \int_{0}^{x-s} \tilde{H}^{\prime}(x-\xi) e^{-\lambda \xi} d \xi+c_{1}+\alpha\left(h-c_{2}\right)-\alpha(h+p) e^{-\lambda x}$ 
We introduce the number $\bar{s}^{*}$ defined by

$$
e^{\lambda \bar{s}^{*}}=\frac{\alpha(h+p)}{c_{1}+\alpha\left(h-c_{2}\right)} .
$$

We can show, just as we had showed $S(\sigma)>\bar{s}$, that $\Sigma(\sigma)>\bar{s}^{*}$.

\subsection{Formulae for $\left(\sigma_{\xi}, s_{\epsilon}, \Sigma_{\varepsilon}, S_{\epsilon}\right)$}

Calling $H_{\sigma}(x)$, the function constructed in the previous section, we can see by the above results that:

$$
\begin{aligned}
& H_{\sigma}(\sigma)-H_{\sigma}(S(\sigma))=\frac{c_{2}+\frac{\alpha h}{1-\alpha}}{\lambda}\left(\frac{c_{1}-c_{2}}{c_{2}+\frac{\alpha h}{1-\alpha}} \log \frac{\alpha(p+h) e^{-\lambda \sigma}-\alpha\left(c_{1}-c_{2}\right)}{X_{0}\left(c_{1}+\frac{\alpha h}{1-\alpha}\right)}\right. \\
& \left.+\frac{1}{1-\alpha}\left[-\log \frac{\left(X_{0}+\alpha\right)\left(c_{1}+\frac{\alpha h}{1-\alpha}\right)}{c_{2}+\frac{\alpha h}{1-\alpha}}+\frac{\left(X_{0}+\alpha\right)\left(c_{1}+\frac{\alpha h}{1-\alpha}\right)}{c_{2}+\frac{\alpha h}{1-\alpha}}-1\right]\right) .
\end{aligned}
$$

We find $\sigma=\sigma_{\epsilon}$ by the equation:

$$
H_{\sigma}(\sigma)-H_{\sigma}(S(\sigma))=K_{2}
$$

and therefore,

$$
\begin{aligned}
& K_{2}=\frac{c_{2}+\frac{\alpha h}{1-\alpha}}{\lambda}\left(\frac{c_{1}-c_{2}}{c_{2}+\frac{\alpha h}{1-\alpha}} \log \frac{\alpha(p+h) e^{-\lambda \sigma}-\alpha\left(c_{1}-c_{2}\right)}{X_{0}\left(c_{1}+\frac{\alpha h}{1-\alpha}\right)}\right. \\
& \left.+\frac{1}{1-\alpha}\left[-\log \frac{\left(X_{0}+\alpha\right)\left(c_{1}+\frac{a h}{1-\alpha}\right)}{c_{2}+\frac{a h}{1-\alpha}}+\frac{\left(X_{0}+\alpha\right)\left(c_{1}+\frac{a h}{1-\alpha}\right)}{c_{2}+\frac{\alpha h}{1-\alpha}}-1\right]\right) .
\end{aligned}
$$

Since the function $H_{\sigma}(\sigma)-H_{\sigma}(S(\sigma))$ is decreasing in $\sigma$, a unique solution of (75) exists provided that $K_{2}$ satisfies the conditions

$$
H_{0}(0)-H_{0}(S(0))>K_{2}>H_{\bar{s}_{\varepsilon}}\left(\bar{s}_{\epsilon}\right)-H_{\bar{s}_{\varepsilon}}\left(S\left(\bar{s}_{\epsilon}\right)\right)
$$

This means that

$$
\begin{aligned}
& \frac{q_{1}}{\lambda}\left(\frac{c_{1}-c_{2}}{q_{1}} \log \frac{\alpha(h+p)}{x_{0} q_{0}}+\frac{1}{1-\alpha}\left[-\log \frac{\left(x_{0}+\alpha\right) q_{0}}{q_{1}}+\frac{\left(x_{0}+\alpha\right) q_{0}}{q_{1}}-1\right]\right) \\
& >K_{2}>\frac{q_{1}}{\lambda(1-\alpha)}\left[-\log \frac{\left(x_{0}+\alpha\right) q_{0}}{q_{1}}+\frac{\left(x_{0}+\alpha\right) q_{0}}{q_{1}}-1\right]
\end{aligned}
$$


where $q_{0}=\left(c_{1}+\frac{\alpha h}{1-\alpha}\right)$ and $q_{1}=c_{2}+\frac{\alpha h}{1-\alpha}$. Once $\sigma_{\epsilon}$ is defined, we can solve (75) to obtain the quantity $X_{0}$ within it and then obtain $s_{\epsilon}, \Sigma_{\varepsilon}$ and $S_{\epsilon}$ explicitly. Specifically the next equation gives $s_{\epsilon}$ in terms of $X_{0}$ and $\sigma_{\epsilon}$.

$$
\left(c_{1}-c_{2}\right)\left(s_{\epsilon}-\sigma_{\epsilon}\right)=K_{2}-\frac{q_{1}}{\lambda(1-\alpha)}\left[-\log \frac{\left(x_{0}+\alpha\right) q_{0}}{q_{1}}+\frac{\left(x_{0}+\alpha\right) q_{0}}{q_{1}}-1\right] .
$$

Then we use the next two equations to obtain $S_{\epsilon}$ and $\Sigma_{\epsilon}$ :

$$
\begin{aligned}
& S_{\epsilon}-s_{\epsilon}=\frac{1}{\lambda(1-\alpha)} \log \frac{\left(x_{0}+\alpha\right) q_{0}}{q_{1}} . \\
& \Sigma_{\epsilon}-s_{\epsilon}=\frac{1}{\lambda(1-\alpha)} \log \left(X_{0}+\alpha\right) .
\end{aligned}
$$

Now the function $H_{\epsilon}(x)=H_{\sigma_{\varepsilon}}(x)$ is defined by the formulae

$$
H_{\epsilon}(x)= \begin{cases}H_{\epsilon}\left(\sigma_{\epsilon}\right), & \forall x \leq \sigma_{\epsilon}, \\ H_{\epsilon}\left(s_{\epsilon}\right)-\left(c_{1}-c_{2}\right)\left(x-s_{\epsilon}\right), & \sigma<x<s_{\epsilon}, \\ H_{\epsilon}\left(s_{\epsilon}\right)+q_{1}\left(x-s_{\epsilon}\right)-\frac{\left(x_{0}+\alpha\right) q_{0}}{\lambda(1-\alpha)}\left(1-e^{-\lambda(1-\alpha)\left(x-s_{\epsilon}\right)}\right), & \forall x \geq s_{\epsilon},\end{cases}
$$

With

$H_{\epsilon}\left(s_{\varepsilon}\right)=\frac{\alpha\left(c_{1}-c_{2}\right)}{\lambda(1-\alpha)}\left(1-e^{-\lambda\left(s_{\varepsilon}-\sigma_{\varepsilon}\right)}\right)$,

and

$H_{\epsilon}\left(\sigma_{\varepsilon}\right)=H_{\epsilon}\left(s_{\varepsilon}\right)+\left(c_{1}-c_{2}\right)\left(s_{\epsilon}-\sigma_{\varepsilon}\right)$

Therefore, the four parameters $\left(\sigma_{\varepsilon}, s_{\varepsilon}, \Sigma_{\varepsilon}, S_{\varepsilon}\right)$ are defined explicitly. We next show the optimality of the $\left(\sigma_{\varepsilon}, s_{\varepsilon}, \Sigma_{\varepsilon}, S_{\varepsilon}\right)$ policy.

\subsection{Optimality of $\left(\sigma_{\varepsilon}, s_{\varepsilon}, \Sigma_{\varepsilon}, S_{\varepsilon}\right)$ Policy}

We begin by verifying the following proposition.

Proposition 5: We have the inequality

$$
\Sigma_{\epsilon}-\sigma_{\varepsilon}<\varepsilon
$$


Proof: From (75), we can write $H_{\epsilon}\left(\sigma_{\varepsilon}\right)<K_{2}+H_{\epsilon}\left(\Sigma_{\varepsilon}\right)$. We use the last equation in (81) to compute

$H_{\epsilon}\left(\Sigma_{\varepsilon}\right)=H_{\epsilon}\left(s_{\varepsilon}\right)+q_{1}\left(\Sigma_{\epsilon}-s_{\varepsilon}\right)-\frac{\left(x_{0}+\alpha\right) q_{0}}{\lambda(1-\alpha)}\left(1-e^{-\lambda(1-\alpha)\left(\Sigma_{\varepsilon}-s_{\varepsilon}\right)}\right)$.

Using (80), and (57), we obtain

$H_{\epsilon}\left(\Sigma_{\epsilon}\right)=H_{\epsilon}\left(s_{\varepsilon}\right)+\frac{\left(x_{0}+\alpha-1\right)}{\lambda(1-\alpha)}\left(c_{2}-c_{1}\right)-K_{1} \frac{q_{1}}{q_{0}}$.

Using (83) we can assert

$$
\begin{gathered}
\left(c_{1}-c_{2}\right)\left(s_{\varepsilon}-\sigma_{\varepsilon}\right)<K_{2}-K_{1}+\frac{\left(c_{1}-c_{2}\right)}{\lambda(1-\alpha)}\left((1-\alpha)\left(\frac{K_{1} \lambda}{q_{0}}+1\right)-X_{0}\right) \\
=K_{2}-K_{1}-\frac{\left(c_{1}-c_{2}\right)}{\lambda(1-\alpha)} \log \left(X_{0}+\alpha\right) .
\end{gathered}
$$

Finally, by using the definition of $\varepsilon$ and (80), we obtain the inequality (84). Then a calculation, which is omitted for brevity, yields the following result:

Theorem 6: Assume

- $\quad(60)$, i.e., $\alpha(h+p)>X_{0} q_{0}$.

- $\quad(76)$, i.e. $H_{0}(0)-H_{0}(S(0))>K_{2}>H_{s_{s}}\left(\bar{s}_{\varepsilon}\right)-H_{s_{s}}\left(S\left(\bar{s}_{\varepsilon}\right)\right)$.

- $\quad \bar{s}_{\varepsilon}+\varepsilon<\bar{s}$.

- $f(\zeta)=\lambda e^{-\lambda \zeta}$. Then the function $H_{\varepsilon}(x)$ defined by (81), with $\sigma_{\varepsilon}$ and $s_{\varepsilon}$ defined by (75) and (78), is the solution of the equation (5) with $\rho(1-\alpha)=g\left(s_{\epsilon}\right)$.

Remark: The structure of extended $(s, S)$ optimal ordering policy is as follows:

- If the beginning inventory level is less or equal to $\sigma_{\varepsilon}$, then order up to $S_{\varepsilon}$.

- If the beginning inventory level is less or equal to $s_{\epsilon}$ but more than $\sigma_{\epsilon}$, then order up to $\Sigma_{\varepsilon}$.

- If the beginning inventory level is more than $s_{\epsilon}$, then do not order.

\section{Conclusion}

In this article, we examined a stochastic inventory model for an infinite horizon problem in which the ordering cost is piecewise linear. We analyzed the model for backlog sales cases. We offered a rigorous mathematical theory and set the conditions which are sufficient to show that $\left(s_{\varepsilon}, S_{\epsilon}\right)$ policy is optimal. The crucial assumption (30) eliminated the possibility that the minimum on the right hand side of equation (11) will be attained by the second term. If we do not make this assumption, this possibility is no longer excluded, leading to an extended optimal policy which is more complex. In this case we showed that a four parameter policy is optimal. Even though this generalization was analyzed only for exponential demand distributions, the theory we provided is constructive and can be extended for general demand distributions. Our theory did not 
rely on $K$-convexity. This approach has the advantage of giving an explicit functional equation for the value function.

Although we have examined this inventory control problem only for a situation with two suppliers, our method is general and can be extended to an arbitrary number of suppliers using a similar approach. Of course, the technical details are accordingly more intricate.

\section{References}

Benjaafar, S.; Chen, D.; Yu, Y. 2018. Optimal policies for inventory systems with concave ordering costs, Naval Research Logistics 65: 291-302.

Bensoussan, A.; Helal, M.A.; Ramakrishna, V.; Sethi, S.P. 2020. Inventory policies for piecewise concave linear ordering cost. Working Paper, The University of Texas at Dallas, USA.

Bensoussan, A. 2011. Dynamic programming and inventory control. Volume 3 of Studies in Probability, Optimization and Statistics, IOS Press. 369p.

Beyer, D.; Sethi, S.P.; Taksar, M. 1998. Inventory Models with Markovian Demands and Cost Functions of Polynomial Growth, Journal of Optimization Theory and Application 98(2): 281-323.

Chen, X.; Simchi-Levi, D. 2012. Pricing and Inventory Management. The Oxford Handbook of Pricing Management. Oxford University Press, 1: 784-824.https:// doi.org/10.1093/oxfordhb/9780199543175.013.0030.

Chen, Y.; Ray, S.; Song, Y. 2005. Optimal pricing and inventory control policy in periodic review systems with fixed ordering cost and lost sales, Naval Research Logist 53(2): 117-136.

Huh. W.; Janakiraman, G. 2008. (s, S) optimality in joint inventory pricing control: an alternative approach, Operations Research 56(3): 783-790.
Karlin, S.; Fabens, A. 1960. The (s,S)-Inventory Model Under Markovian Demand Process. In Mathematical Methods in the Social Sciences, Edited by K. Arrow, S. Karlin and P. Suppes, Stanford University Press, Stanford, California, 159-175.

Ozekici, S.; Parlar, M. 1995. Periodic review inventory models in random environments. Working Paper, McMaster University, Hamilton, Ontario.

Porteus, E.L. 1971. On the optimality of generalized ( $s$, S) Polices, Management Science 17: 411-426.

Scarf, H. 1960. The Optimality of $(S, s)$ Policies in the Dynamic Inventory Problem. Mathematical Methods in the Social Sciences, Stanford University Press, Stanford, CA, 196-202.

Sethi, S.P.; Cheng, F. 1997. Optimality of $(s, S)$ Policies in Inventory Models with Markovian Demand Processes, Operations Research 45(6): 931-939.

Singha, K.; Buddhakulsomsiri, J.; Parthanadee, P. 2017. Mathematical Model of ( $R, Q)$ Inventory Policy under Limited Storage Space for Continuous and Periodic Review Policies with Backlog and Lost Sales, Mathematical Problems in Engineering 2017: 1-9, Article ID 4391970.

Song, J.S.; Zipkin, P. 1993. Inventory Control in a Fluctuating Demand Environment, Operations Research 41(2): 351-370.

Song, Y.; Ray, S.; Boyaci, T. 2009. Optimal dynamic joint inventory-pricing control for multiplicative demand with fixed order costs and lost sales, Operations Research 57(1): 245-250.

Veinott, Jr. A.F. 1966. On the Optimality of $(s, S)$ Inventory Policies: New Conditions and a New Proof, SIAM Journal on Applied Mathematics 14(5): 10671083. 